\title{
Multi-Agent Collaboration in Competitive Scenarios
}

\author{
Florian Fuchs \\ Technische Universität München, 80290 München, Germany \\ phone: $++49-8948095$ 254, fax: $++49-8948095250$ \\ email: fuchsf@informatik.tu-muenchen.de
}

\begin{abstract}
For many multi-agent scenarios one can assume that the agents behave cooperatively and contribute to a common goal according to their design. However, our work focuses on competitive scenarios which are characterized by the agents' strong local interests, their high degree of autonomy, and the lack of global goals. Therefore, two agents will cooperate if, and only if, both will gain - or at least expect to gain - from that cooperation.

This paper presents a conflict resolution mechanism which is appropriate for competitive resource allocation in dynamic environments which is based on compromising. It integrates a goal relaxation mechanism, negotiation histories, and multilateral negotiations.
\end{abstract}

\section{Keywords}

Agents, non-cooperative, collaboration, compromise

\section{INTRODUCTION}

In contrast to multi-agent settings which follow a fully cooperative model, there are scenarios with quite different qualities. These are settings without a global goal function and with agents which follow exclusively local goals. Cooperation between two agents takes place only if both sides expect to profit. We denote such scenarios as competitive.

A restricted view to the global system state and agents pursuing different local goals are typical qualities in multi-agent systems, whereas in traditional centralized structured approaches these qualities hardly play any role. As a consequence of these problem characteristics conflicts arise among the agents which have to be resolved. I.e. mechanisms for conflict resolution have to be integrated into a multi-agent approach in order to obtain global consistent solutions.

In competitive scenarios some problems make the conflict resolution more difficult than in fully cooperative ones. First of all, the amount of common knowledge is very 
small. In general, agents do not know the other agents' plans, goals, strategies, etc. An agent will not provide the others with that kind of information to avoid that they can take advantage of this knowledge. Thus, all the agents can do is to extract information out of the negotiation processes and build models of its competitors which are largely uncertain.

Furthermore, if an agent provides some information, it may not tell the truth. Interesting questions are: When is it profitable for agents to lie, and how can lies be discouraged. Palatnik and Rosenschein investigated some problems arising in that context [PR94].

The resolution of a conflict is achieved by the application of a conflict resolution strategy. There are different situations in which conflicts occur, e.g. the conflict may concern a part of the schedule which is either still coarse grained or where already much fine planning was involved. Furthermore, we have different scenarios, e.g scenarios with many alternative resources or such without any alternatives. Different situations and different problem scenarios require varying strategies to resolve a conflict successfully. An agent therefore needs conflict resolution knowledge that enables it to react appropriately when it is faced with a conflict.

This paper presents a conflict resolution mechanism for distributed resource allocation which is suitable for competitive scenarios. The rest of the paper is structured as follows: In section 2 requirements for an appropriate conflict resolution mechanism are pointed out. Section 3 is dedicated to our application scenario and section 4 introduces our compromising concept, and finally, in section 5 some conclusive remarks are made.

\section{COMPETITIVE AGENTS}

Competitive scenarios show some specific characteristics which require appropriate conflict resolution strategies. Most methods suitable for fully cooperative settings are not applicable.

Many approaches to conflict resolution among cooperative agents employ a more or less static set of strategies, one of which is chosen as a common strategy in the case a conflict occurs. This strategy might be either chosen through a negotiation between the agents (see for example the Cooperative Experts Framework [LLC91]), or might be agreed upon directly because the agents have identical conflict resolution units (see for example the Cooperative Design Engine [KB91]). With strategies which are common to all agents it is virtually impossible to model competitive scenarios where the agents are not willing to share the knowledge about their employed strategies. They have to be enabled to apply arbitrary strategies, however they believe their local interests are supported efficiently with.

Another popular method in cooperative domains is to let one of the conflicting parties play a special role in the conflict resolution process. In the work of Polat and Guvenir, for instance, the agents have different roles in the negotiation process according to their knowledge and problem-solving capabilities [PG92]. As far as competitive scenarios are concerned, this is not a feasible way. Here, agents will refuse to take part in negotiations in which they do not have equal rights, and therefore, may be put at a disadvantage or may be cheated. 
Quite similar drawbacks have approaches in which the conflict resolution is done by an arbitrator agent (see for example [Wer90] or [SRSF90]). The existence of such an arbitrator is a quite unrealistic assumption in most cases. Conflicts rather have to be resolved by direct negotiation between the conflicting parties.

Let us briefly summarize the requirements for a conflict resolution mechanism suitable for our scenario which we have found so far:

\section{- Symmetry}

None of the conflicting parties must play an extraordinary role in the conflict resolution process, they must be given equal rights.

\section{- Private Strategies}

Strategies have to be private in the sense that they are freely choosable by each of the conflicting parties, and they are not accessible by the other agents.

\section{- No Arbitrators}

The agents have to resolve conflicts without help from some higher level instance.

Furthermore, there are several requirements which are not specific for competitive scenarios, but have to be considered in other settings as well. The most important ones are listed in the following:

\section{- Stability}

The conflict resolution should not yield an unstable behavior of the overall system, since instability results in undesired unpredictability of future market trends.

\section{- Adaptability}

An important characteristic of a market scenario is its dynamic, the agent's goals are subject to constant change. Therefore, an agent must have the capability to choose appropriate strategies, taking into consideration its own goals, the model it has about its negotiation partners, as well as the needs of its clients.

\section{APPLICATION SCENARIO}

The environment in which our agents are situated is a scenario from the field of distributed resource allocation. Orders which are brought into the system from extern clients have to be assigned to resources under consideration of temporal aspects. Therefore, the scenario includes a distributed scheduling problem, which is to be solved by competitive agents. The participating agents try to influence those parts of the global schedule positively with respect to their local optimality criteria which are interacting with their local plans.

We are treating only worth-oriented domains which allow for a partial satisfaction of goals, and therefore, for a compromise in the case of goal conflicts. This stands in contrast to task-oriented or state-oriented domains, where one of the conflicting parties has to refrain from its goal totally in the case of a conflict. 


\subsection{Scheduling model}

The employed scheduling model is similar to what is proposed in [Win93]. This extended job shop comprises a set of resources which is divided into resource groups corresponding to certain activities. Then there is a set of tasks aggregated to jobs by means of a partial order. The model allows that jobs are brought into the system dynamically (online scheduling). This model is characterized in detail in [Fuc96].

Schedules are not represented by means of exact time intervals or time points. Despite, density functions over time are used for each resource. A density function serves as a coarse grained representation of the capacity usage of a resource and provides flexibility with respect to the actual start points of intervals. They allow for an efficient evaluation of resource requests. Dealing with exact schedules would be virtually impossible considering the early planning state, and therefore, the high planning uncertainty at this planning level.

\subsection{Agent model}

This section gives an overview of the applied agent model. Therefore, we will describe our agent design and how the agent is situated within its environment. Figure 1 shows the conceptual structure of the agent model which is used in our cooperation scenario. The agent is embedded in its environment which consists of three parts: The client environment from where jobs are brought into the system, the execution environment functioning as an outlet for jobs, and finally the agent environment where jobs may be distributed via some cooperation mechanisms.

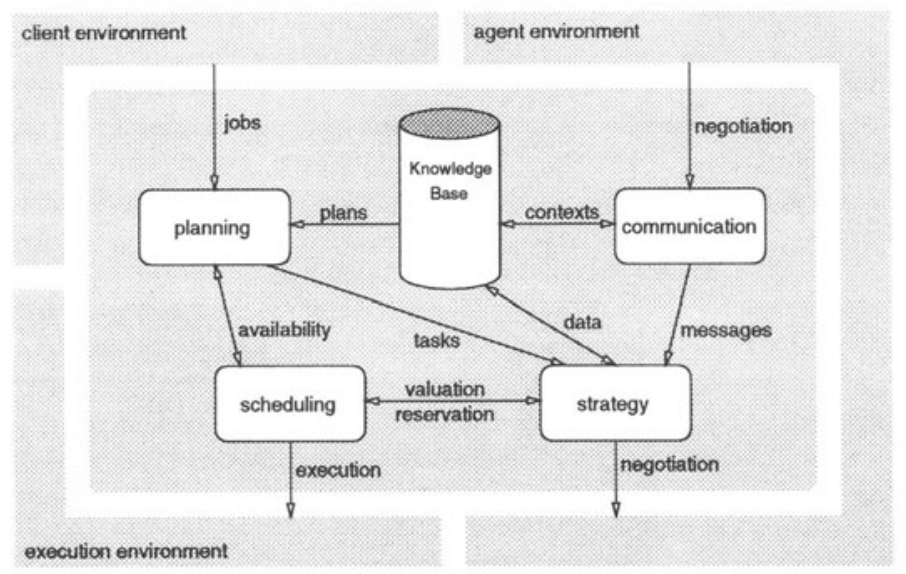

Figure 1: agent architecture

An agent encapsulates all the knowledge it needs to realize its goals and also the appropriate processing mechanisms for that knowledge. The knowledge is kept explicitly 
represented in a local knowledge base which contains in the first place knowledge about other agents, the own internal state, and skeleton plans that are instructions on how to process jobs.

In addition to the knowledge base there are four agent components which operate quite independently and are connected via some internal interfaces.

The planning unit maintains a set of jobs it receives in irregular intervals from some clients. The planning unit has to build a production plan for a job - mostly by getting a prebuilt plan from the knowledge base - and determines a task which is to be scheduled next.

An agent is able to communicate through its communication unit with other agents. From a received message it extracts information and updates its local knowledge base accordingly. Furthermore, the message is put into a certain context according to its type and contents. The agent then changes to the role intended for this context.

The strategy unit contributes the strategic part to the conflict management. This unit is responsible for generating an appropriate response to a received message (see section 3.3).

The scheduling unit manages schedules and evaluates proposals for resource allocations from the strategy unit. This unit is also the one which is connected to the execution environment and makes the necessary steps for the execution of tasks.

\subsection{Market System}

The basis of our cooperation model is a market-oriented system (cf. the notions computational ecology [HH88], agoric open systems [MD88], and market-oriented programming [Wel93]). With market-oriented system we understand a system which provides market mechanism like a pricing system or negotiation platforms which can be used by the participants of the market.

Those participant are agents. They own resources which are necessary for processing the jobs. Generally, not all resources necessary for all steps of a job are available locally. Therefore, an appropriate decomposition and distribution of jobs is essential.

For this reason, a pricing mechanism is introduced as follows. Each resource causes costs. This is especially true for the time a certain activity is processed. But also resources lying idle are not free of costs. All those costs have to be payed by the respective owner of a resource. Whenever a job is given out to some supplier this supplier will pass on the costs to its client. An additional fee is charged for the usage of a resource what allows for making some profit.

\section{CONFLICT RESOLUTION}

Different local interests yield inevitably goal conflicts which are eliminated by mutual goal relaxation - a compromise. This goal relaxation and the necessary negotiation is discussed in detail in the following two sections. 


\subsection{Negotiation}

The allocation of resources is subject to negotiation. A negotiation is characterized by a negotiation protocol describing a set of negotiation primitives as communicable messages and all valid sequences of those primitives of a negotiation.

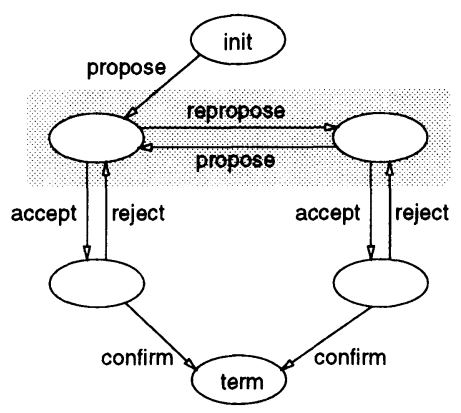

Figure 2: protocol

The negotiation protocol which is made use of is an extended version of the well-known contract net protocol [Smi88]. The four phases request, offer, order, and confirmation are replaced with an embedding of propose and repropose (see figure 2) - necessary to enable the compromising mechanism.

The contents of these primitives is taken as a proposal for a contract (draft contract), which includes two parts: First, a specification of the object of the contract - mainly the resource in question - and second, negotiable terms of the contract, for example the allocation time interval or the price for the allocation. During a negotiation the agents may either agree on the terms and thus make a contract, or the negotiation fails because the local interests are incompatible.

At each negotiation at least one supplier and one client takes part. Supplier and client are roles the agents play temporarily.

\subsection{Compromising}

The agents are characterized by a rational behavior. Rationality is given when an agent adopts the goal to increase its own profit which is defined by means of its local utility function.

The utility an agent gets from a contract is influenced mainly by two aspects. On the one hand costs arise from what an agent is obliged to by a contract. The client is obliged to pay the negotiated price and the supplier has to provide resources. On the other hand a contract has a certain worth for both parties. The supplier gets the negotiation price and the client is given resources and thus the possibility to fulfill its due dates. The resulting utility is the difference of worth and costs.

There are two more points, which are important for an agent's utility. Resources do not only cause costs when they are allocated but also when they lye idle. And furthermore, 
for exceeding due dates some kind of punishment is introduced. Both points serve as an incentive for the agents to take part in negotiations and show willingness to compromise.

Costs and worth of a draft contract depend on the current schedule and the quality of the resulting schedule with respect to the preferences of an agent, i.e. with respect to capacity usage, flow time, job tardiness, etc. If an agent was alone in the society, it could build the schedule that has maximum worth and therefore, the maximum utility. Otherwise, if it is in a society of competitors, goal conflicts are inevitable and compromises concerning the schedule have to be found. The market-oriented system serves as a tool to reach those compromises.

For achieving a compromise between two agents a mutual modification of a draft contract takes place. Such a modification is like the search in a search space which is defined by the terms of the contract. Each point in the search space corresponds exactly to one possible draft contract. The search for a counterproposal is guided by a evaluation function which assigns an evaluation to every draft proposal. This evaluation depends on the current schedule and state of the negotiation, i.e. especially the current terms of the contract.

The following three aspects are considered through the evaluation function and are weighted appropriately:

\section{- local goals}

An agent's local goals slip into the evaluation by means of the agent's utility.

\section{- negotiation history}

An agent tries to extract information out of its encounters with other agents and to use this information in future encounters. Therefore, it finds out the flexibility of its competitors in negotiation concerning each single term of a contract. It uses these flexibility values to guide the search for a counter proposal into a direction which is acceptable for its competitor.

\section{- time pressure}

An agent is faced with two kinds of time pressure during a negotiation. First, it has to prevail over its competitors and is therefore forced to come to a acceptable proposal in reasonable time. Second, there are time constraints which result from given due dates. For these reasons, the evaluation of a draft contract is corrected appropriately depending on the expired negotiation time.

In the resulting search space one of the standard search algorithms - e.g. simulated annealing - can be applied to generate a counter proposal. The termination of this process is guaranteed because the utility of a contract increases when the probability of exceeding a due date becomes higher. The convergence of this process can be controlled by the agents by means of giving the time pressure aspect more or less weight in their evaluation.

Figure 3 shows an example for typical evaluations which are extracted from a test run with a given planning state. For visualization reasons just two terms of a contract are displayed. One can see clearly the contradictionary interests of supplier and client concerning the price. 

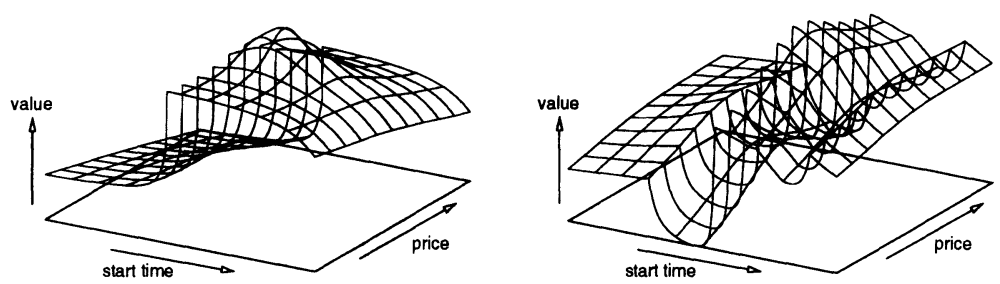

Figure 3: example for proposal evaluations of client (left) and supplier (right)

\section{CONCLUSION AND FUTURE WORK}

This paper presented a conflict resolution concept for multi-agent systems which is suitable for competitive scenarios which is based on compromising.

The most important feature of this concept is that it follows strictly the non-cooperation paradigm. It integrates aspects which are realistic for that kind of scenarios, goal relaxation, uncertain knowledge, negotiation histories, and multilateral negotiations. The agents' conflict resolution strategies are private, i.e. not accessible by other agents. Therefore, an agent is enabled to preserve its local interests. Of course the possibility to share a common strategy remains.

Currently effort is spent on finishing a prototypical implementation of the described system. This prototype will also include a simulation environment which serves as a testbed for the efficiency of different strategies in different manufacturing scenarios. The next goal is to investigate the effects of some strategies on the scheduling results - especially the impact on job lateness, in-process inventory, job idle time, and machine utilization. We hope to finish the first prototype implementation in a few months and will come out with the first experimental results soon afterwards.

\section{References}

[Fuc96] F. Fuchs. Multi-Agent Collaboration in Competitive Scenarios. In Proc. of the $15^{\text {th }}$ Workshop of the UK Planning and Scheduling Special Interest Group, Liverpool, 1996.

[HFL96] S. Hahndel, F. Fuchs, and P. Levi. Distributed Negotiation-based Task Planning for a Flexible Manufacturing Environment. In J. W. Perram and J.-P. Müller, editors, Distributed Software Agents and Applications, number 1069 in Lecture Notes in Artificial Intelligence. Springer, 1996. 
[HH88] B. A. Huberman and T. Hogg. The Behaviour of Computational Ecologies. In B. A. Huberman, editor, The Ecology of Computation, number 2 in Studies in Computer Science and Artificial Intelligence, pages 77-115. North-Holland, 1988.

[KB91] M. Klein and A. B. Baskin. A Computational Model for Conflict Resolution in Cooperative Design Systems. In S. M. Deen, editor, CKBS'90: Proc. of the International Working Conference on Cooperating Knowledge Based Systems, October 1990, Univ. of Keele, UK, pages 201-219. Springer, Berlin, 1991.

[LLC91] S. Lander, V. R. Lesser, and M. E. Connell. Conflict Resolution Strategies for Cooperating Expert Agents. In S. M. Deen, editor, CKBS'90: Proc. of the International Working Conference on Cooperating Knowledge Based Systems, October 1990, Univ. of Keele, UK, pages 183-200. Springer, Berlin, 1991.

[MD88] M. S. Miller and K. E. Drexler. Markets and Computation: Agoric Open Systems. In B. A. Huberman, editor, The Ecology of Computation, number 2 in Studies in Computer Science and Artificial Intelligence, pages 133--176. NorthHolland, 1988.

[PG92] F. Polat and H. A. Guvenir. A Conflict Resolution Based Cooperative Distributed Problem Solving Model. In Proc. of AAAI-92, pages 106-115, 1992.

[PR94] M. Palatnik and J. S. Rosenschein. Long Term Constraints in Multiagent Negotiation. In $13^{\text {th }}$ International DAI Workshop, pages 265-279, Seattle, Washington, 1994.

[Smi88] R. G. Smith. The Contract Net Protocol: High-Level Communication and Control in a Distributed Problem Solver. In A. H. Bond and L. Gasser, editors, Readings in Artificial Intelligence. Morgan Kaufmann Publishers Inc., San Mateo, California, 1988.

[SRSF90] K. Sycara, S. Roth, N. Sadeh, and M. Fox. Managing Resource Allocation in Multi-Agent Time-Constrained Domains. In Proc. of a Workshop on Innovative Approaches to Planning, Scheduling, Control. Kaufmann, 1990.

[Wel93] M. P. Wellman. A Market-Oriented Programming Environment and its Application to Distributed Multicommodity Flow Problems. Journal of Artificial Intelligence Research, 1:1-23, 1993.

[Wer90] K. Werkman. Design and fabrication problem solving through cooperative agents. Technical report, Lehigh University Bethlehem, 1990.

[Win93] A. Winkelhofer. Zeitrepräsentation und merkmalsgesteuerte Suche zur Terminplanung. PhD thesis, Technische Universität München, 1993. 